\title{
Sincronização de um Modelo Metapopulacional com Taxa de Reprodução Intrínseca Dependente do Tempo
}

\author{
Carlos E. Espinosa, \\ Programa de Pós-Graduação em Engenharia Mecânica, UFRGS \\ 90050-170, Porto Alegre, RS \\ E-mail: eduardo.espinosa@ufrgs.br \\ Jacques A. L. da Silva \\ Programa de Pós-Graduação em Mátemática Aplicada, UFRGS \\ 91509-900, Porto Alegre, RS \\ E-mail: jaqx@mat.ufrgs.br
}

\begin{abstract}
Resumo: $O$ estudo de sincronização de sistemas metapopulacionais desperta interesse dos estudiosos na área de biologia matemática, uma vez que é a partir da sincronização que podemos avaliar e prever o risco da extinção de espécies. Este presente trabalho propõe estudar os fatores que causam o fenômeno da sincronização de um modelo metapopulacional com taxa de reprodutividade intrínseca dependente do tempo. Apresentamos condições que o sistema deva satisfazer para obtermos sincronização, a qual é determinada por dois parâmetros distintos: o número de Lyapunov, que está relacionado à dinâmica local, e Lambda, que está relacionado à migração. O produto desses dois parâmetros estabelece um critério para estabilidade local assintótica de órbitas caóticas, possibilitando ou não a sincronia do sistema. Apresentamos resultados numéricos afim de verificar o critério analítico para a sincronização.
\end{abstract}

Palavras-chave: Metapopulação, Sincronização, Extinção, Estabilidade Local Assintótica

\section{Exposição e formulação problema}

Considere $n$ sítios numerados de 1 a $n$. Em cada um desses sítios existe uma população de uma única espécie a qual denotamos de população local, ou subpopulação. Esses sítios estão cercados por um ambiente hostil e inadequado para a sobrevivência e persistência da espécie. A cada geração (a cada passo de tempo), essas populações passam por dois processos distintos: a dinâmica local, composta pela reprodução e sobrevivência e a dispersão ${ }^{1}$ (migração). Supomos que a dinâmica local precede a migração.

A taxa de reprodução intrínseca $r$ depende do tempo, ou seja, $r_{t+1}=g\left(r_{t}\right)$, onde $r$ é a taxa de reprodutividade intrínseca da população e $g$ é uma função suave dada. Logo, a função $f$ que incorpora os processos de reprodução e sobrevivência possui duas variáveis dependentes do tempo, a saber, $x$ e $r$, e é definida como

$$
x_{t+1}=f\left(x_{t} ; r_{t}\right)=x_{t} e^{r_{t}\left(1-x_{t}\right)},
$$

$t \geq 0, i=1, \ldots, n$, onde $f$ representa uma função suave definida em $[0, \infty)$.

Após transcorrido o processo de reprodução e sobrevivência, temos o início do processo migratório. A cada tempo $t$, após a dinâmica local, uma fração $\mu$ de indivíduos deixa um dado sítio e migra para outros sítios mais próximos. Trabalharemos com a migração independente da densidade tal que $0 \leq \mu \leq 1$.

\footnotetext{
${ }^{1}$ Ambas as palavras migração e dispersão serão denotadas para descrever os movimentos de um sítio para o outro, portanto, não há diferença entre elas.
} 
Consideramos $C=\left[c_{j i}\right]$ a matriz de acoplamento dos sítios, onde $c_{j i}$ indica a proporção de indivíduos que sai do sítio $i$ e chega no sítio $j$. Supomos que a matriz $C$ é duplamente estocástica (o que reflete o fato de não haver morte durante o processo migratório). Assumimos ainda que $c_{i i}=0$ (não há migração para o próprio sitio). Desta forma a dinâmica da metapopulação é dada por

$$
G\left(X_{t}\right)=x_{t+1}^{i}=(1-\mu) f\left(x_{t}^{i} ; r_{t}\right)+\sum_{j=1}^{n} c_{i j} \mu f\left(x_{t}^{j} ; r_{t}\right),
$$

para $i, j=1, \ldots, n$.

O primeiro termo do lado direito da equação (2) representa os indivíduos que permanecem no sítio $i$ no tempo $t$, enquanto que o segundo termo representa a soma de todos os imigrantes do sítio $i$. Para evitar efeitos de fronteira, trabalhamos com condições de contorno periódicas em formas de anéis cíclicos. Quando só há migração para os dois vizinhos adjacentes ao sítio $i$, denotamos conexão local. Quando temos migração para todos os sítios vizinhos ao sítio $i$, dizemos que a conexão é global. Padrões de migração realísticos encontram-se entre a conexão global e local [1]. A topologia da rede se dará somente através de anéis cíclicos de conexão global, uma vez que para conexão local os sítios não sincronizam para nenhum valor de função migratória $\mu[2]$.

Dinâmicas síncronas ocorrem quando todos os sítios com condições iniciais diferentes, apresentam o mesmo número de indivíduos a cada tempo $t$. Uma sincronização é perfeita ou total se todos os sítios apresentarem a mesma densidade populacional em cada tempo $t$, isto é,

$$
x_{t}^{j}=x_{t}^{i}=x_{t},
$$

$i, j=1, \ldots, n$.

Matematicamente, sincronização significa que a dinâmica do sistema está restrita a um subespaço invariante. Uma órbita iniciada nessa reta, permanece nela ao longo do tempo. Nosso objetivo é analisar a estabilidade local assintótica das soluções sincronizadas, ou seja, determinaremos se órbitas que iniciam próximas do estado sincronizado serão atraídas ou repelidas para esse estado.

\section{Resultados Obtidos}

Para analisar a estabilidade local assintótica, vamos decompor $\mathbb{R}^{n}$ em um espaço de dimensão 1 o qual se restringe ao movimentos síncronos e $n-1$ subespaços que são transversais a esse subespaço. Para desenvolver o critério de estabilidade, faremos a perturbação transversal desses subespaços transversais tenderem a zero.

Considere $X_{t}=\left(x_{t}^{1}, \ldots, x_{t}^{n} ; r_{t}\right)$. Definimos por $X_{t}^{*}=\left(x_{t}, \ldots, x_{t} ; r_{t}\right)^{T} \in \mathbb{R}^{n}$ uma órbita sincronizada . É facil ver que ao aplicarmos a órbita sincronizada no sistema (2) obtemos

$$
x_{t+1}=f\left(x_{t} ; r_{t}\right),
$$

$i=1, \ldots, n, \forall t$. Logo, a dinâmica de cada sítio no estado sincronizado satisfaz a dinâmica de um sítio isolado.

A Jacobiana associada ao sistema (2) é dada por

$$
J\left(X_{t}\right)=\frac{\partial G\left(X_{t}\right)}{\partial\left(x_{t}^{1}, \ldots x_{t}^{n}\right)} .
$$

De (5), podemos definir

$$
f^{\prime}\left(x_{t} ; r_{t}\right)=\frac{\partial f\left(x_{t} ; r_{t}\right)}{\partial x_{t}},
$$

ou seja, derivamos $f$ somente em relação a $x$, e não a $r$. 
Para analisar a estabilidade local assintótica das soluções sincronizadas, linearizamos o sistema (2), dado por $X_{t+1}=G\left(X_{t}\right)$, em torno da órbita sincronizada, e obtemos

$$
\Delta_{t+1}=J\left(X_{t}^{*}\right) \Delta_{t}
$$

onde $J\left(X_{t}^{*}\right)$ é a Jacobiana do sistema aplicada na órbita síncrona e $\Delta_{t}$ representa uma pequena perturbação do estado sincronizado. A matriz Jacobiana aplicada na órbita sincronizada é dada por

$$
J\left(X_{t}^{*}\right)=f^{\prime}\left(x_{t} ; r_{t}\right)(I-\mu B),
$$

onde $I$ é a matriz identidade e $B=I-C$.

Notamos que $\lambda=0$ é um autovalor de $B$ associado ao autovetor $v=(1,1, \ldots, 1)^{T}$, e portanto, $\lambda=1$ é um autovalor de $I-\mu B$ associado ao autovetor $v=(1,1, \ldots, 1)^{T}$. Pelo teorema de Gershgorin [2], $\lambda=1$ é autovalor dominante de $I-\mu B$.

Sem perda de generalidade, supomos que na conexão entre os sítios não exista nenhum conglomerado isolado, isto é, que a matriz $C$ é irredutível ${ }^{2}$. Pelo Teorema de Perron-Frobenius [2], a matriz irredutível $C$ possui $\lambda=1$ como seu autovalor simples associado ao autovetor $v=(1,1, \ldots, 1)^{T}$. Com isso, $\lambda=0$ é autovalor simples da matriz $B$. Então existe uma base no $\mathbb{R}^{n}$ na qual a transformação linear dada por $B$ assume uma forma de matriz diagonal em bloco. A matriz $B$ pode ser representada como:

$$
B=Q\left[\begin{array}{ccc}
0 & \ldots & 0 \\
\vdots & & \\
0 & & A
\end{array}\right] Q^{-1}
$$

onde $A$ é uma matriz $(n-1) \times(n-1)$ e $Q$ é uma matriz mudança de base apropriada. Portanto,

$$
I-\mu B=Q\left[\begin{array}{cccc}
1 & 0 & \ldots & 0 \\
0 & & \\
\vdots & & \\
0 & I-\mu A &
\end{array}\right] Q^{-1}
$$

possui um autovalor $\lambda=1$ que está associado ao autovetor $v=(1, \ldots, 1)^{T}$ o qual é diagonal do espaço de fase, isto é, é o subespaço invariante que se restringe ao movimentos síncronos. Qualquer perturbação poderá ocorrer nesse subespaço que ainda assim continuará no mesmo. Entretanto, as perturbações em $I-\mu A$ são transversais a diagonal de fase e deverão tender a zero para que possamos verificar a estabilidade do estado síncrono.

Com a finalidade de estudar o crescimento das perturbações transversais á órbita sincronizada, consideramos

$$
J\left(X_{t}\right)=Q f^{\prime}\left(x_{t} ; r_{t}\right)(I-\mu A) Q^{-1}
$$

e analisamos a evolução da componente transversal do mesmo

$$
\Delta_{t+1}=f^{\prime}\left(x_{t} ; r_{t}\right)(I-\mu A) \Delta_{t}
$$

onde $\Delta$ é um vetor perturbação no $\mathbb{R}^{n-1}$.

A análise da estabilidade de (12) é feita utilizando a teoria dos expoentes de Lyapunov [3]. Utilizando uma técnica que desacopla o sistema (7), supondo $A$ diagonalizável e fazendo uma mudança de variável $\Delta=S \bar{\Delta}$ (ver em Espinosa [2]), obtemos

$$
\bar{\Delta}_{t+1}=f^{\prime}\left(x_{t} ; r_{t}\right)(I-\mu D) \bar{\Delta}_{t}
$$

\footnotetext{
${ }^{2}$ Uma matriz não-negativa é dita redutível se existir uma partição do conjunto de índices $1, \ldots, n$ em conjuntos disjuntos não-vazios $I_{1}$ e $I_{2}$ tal que $a_{i j}=0$ toda a vez que $i \in I_{1}$ e $j \in I_{2}$. Caso contrário, a matriz é dita irredutível.
} 
onde $D$ é a matriz diagonal dos autovalores de $A$. Portanto, afim de encontrarmos a estabilidade transversal da órbita sincronizada, temos que a perturbação transversal se aproximará de zero, se e somente se

$$
\lim _{t \rightarrow \infty}\left\|Q_{t-1} \ldots Q_{1} Q_{0}\right\|^{1 / t}<1
$$

onde $Q_{t}=(I-\mu D) f^{\prime}\left(x_{t} ; r_{t}\right)$, para todos autovalores da matriz $A$. Deste modo, as perturbações transversais ao subespaço invariante tendem a zero. Lembramos que as perturbações paralelas podem ocorrer livremente pois sempre estarão no estado síncrono.

Temos que

$$
\begin{aligned}
\left\|Q_{t-1} \ldots Q_{1} Q_{0}\right\| & =\left\|(I-\mu D) f^{\prime}\left(x_{t-1}\right) \ldots(I-\mu D) f^{\prime}\left(x_{1}\right)(I-\mu D) f^{\prime}\left(x_{0}\right)\right\| \\
& =\left\|(I-\mu D)^{t}\right\| \prod_{t=0}^{t-1}\left|f^{\prime}\left(x_{t}\right)\right| .
\end{aligned}
$$

Portanto,

$$
\lim _{\tau \rightarrow \infty}\left\|Q_{\tau-1} \ldots Q_{1} Q_{0}\right\|^{1 / \tau}=L\left(x_{0} ; r_{0}\right) \Lambda
$$

onde

$$
L\left(x_{0} ; r_{0}\right)=\lim _{\tau \rightarrow \infty} \prod_{t=0}^{\tau-1}\left|f^{\prime}\left(x_{t} ; r_{t}\right)\right|^{1 / \tau}
$$

é o número de Lyapunov com órbita iniciando em $x_{0}$ e com taxa de reprodutividade inicial $r_{0}$, enquanto $\Lambda$ é dado por

$$
\Lambda=\max _{i=2, \ldots, n}\left(\left|1-\mu \lambda_{i}\right|\right) .
$$

Para cada $i=2, \ldots, n, L\left(x_{0} ; r_{0}\right)\left|1-\mu \lambda_{i}\right|$ representa um número de Lyapunov Transversal, e $i=1$ representa o número de Lyapunov Paralelo, que somado com os anteriores resultam nos $n$ números de Lyapunov associados ao sistema (2). Portanto se quisermos obter estabilidade assintótica consideramos apenas o maior valor do número de Lyapunov transversal, uma vez que este corresponde à direção da maior expansão $L\left(x_{0} ; r_{0}\right) \Lambda>1$ ou menor contração $L\left(x_{0} ; r_{0}\right) \Lambda<1$ das órbitas [2].

A menos de conjuntos de medida $\rho$ nula, podemos eliminar a dependência do Número de Lyapunov em $x_{0}$ e estabelecer o critério para estabilidade local assintótica:

$$
\begin{aligned}
& L \Lambda>1 \Rightarrow \text { Instabilidade Local do Estado Síncrono } \\
& L \Lambda<1 \Rightarrow \text { Estabilidade Local do Estado Síncrono }
\end{aligned}
$$

Na expressão acima, quando calculamos $L$, se optarmos por retirar a dependência da condição inicial $r_{0}$, precisamos integrar $L$ com respeito a medida que deixa a função $g$ invariante. Com isso, a expressão que é representada por um somatório de $t$ dependendo de $r_{0}$, vira uma integral agora independendo da condição inicial. O processo é análogo para a remoção da dependência na condição inicial de $x_{0}$ da função $f$. Logo, teremos uma integral dupla para o cálculo de $L$ (ver Espinosa [2]). Entretanto, o cálculo de $\Lambda$ não pode ser expresso por uma integral, uma vez que não há variáveis independentes para a equação (18).

Para resultados mais gerais, pode ser um tanto complicado trabalhar com essas definições de $L$ sem um prévio conhecimento das medidas naturais de probabilidade $\rho$ e $\delta$. As medidas $\rho$ e $\delta$ normalmente trazem características singulares como em Eckman e Ruelle [3].

\section{Simulações numéricas}

Calculamos os Números de Lyapunov Transversais do sistema (2) onde a função $f\left(x_{t} ; r_{t}\right)$ representa a dinâmica local definida na equação (1), e $0 \leq \mu \leq 1$.

As condições iniciais do sistema são escolhidas aleatoriamente próximas ao estado síncrono, ou seja,

$$
X_{0}=\left(x_{0} \pm 0.01 \epsilon_{1}, x_{0} \pm 0.01 \epsilon_{2}, \ldots, x_{0} \pm 0.01 \epsilon_{n}\right),
$$


onde $X_{0} \in \mathbb{R}^{n}, x_{0}=1$ é o ponto de equilíbrio homogêneo não-trivial do sistema e $\epsilon_{i}, i=1, \ldots, n$, são valores aleatórios escolhidos entre $[-1,1]$.

Calculamos numericamente Lambda $(\Lambda)$ através da equação

$$
\Lambda=\max _{j=2, \ldots, n}\left|1-\mu \lambda_{j}\right|
$$

onde $\lambda_{j}$ são os autovalores da matriz $B$, e o número de Lyapunov $L$ é dado pela seguinte expressão

$$
L\left(x_{0} ; r_{0}\right)=\lim _{\tau \rightarrow \infty} \prod_{t=0}^{\tau-1}\left|f^{\prime}\left(x_{t} ; r_{t}\right)\right|^{1 / \tau}
$$

Como estamos interessados em analisar a extinção da metapopulação, que está relacionada com a sincronização de órbitas caóticas, utilizamos valores de $r$ que façam a dinâmica local gerar oscilações caóticas, não apenas escolhendo $r>2.5$, mas também dando atenção para não escolhermos valores que se encontrem nas janelas de periodicidade. Portanto, estamos interessados apenas onde a função comporta-se de forma caótica, que segundo o critério mostrado anteriormente, será o comportamento de toda a metapopulação em caso de sincronia.

Para cálculo de $L$, neste caso em que $r$ depende do tempo, foram escolhidas medidas de probabilidade naturais.

Para a distribuição dos valores em $r$ foram escolhidas 5 medidas a serem usadas como exemplos, a medida de Dirac no ponto $r=r_{0}$, a medida de probabilidade centrada em dois pontos $r_{1}$, $r_{2}$ (período 2), a medida de probabilidade centrada em quatro pontos $r_{1}, r_{2}, r_{3}$ e $r_{4}$ (período 4), a medida de probabilidade distribuída contínua e uniformemente num intervalo $[a, b]$ e a medida de distribuição de Gauss no intervalo $[0, \infty)$.

O critério para a comparação se dará do seguinte modo:

- Tomamos um $r_{0}$, o qual utilizamos na distribuição de Dirac. Escolhemos $r_{0}$ de tal forma que faça o sistema oscilar de de forma caótica;

- Para a distribuição centrada em dois pontos, escolhemos $r_{1}=r_{0}-\beta, r_{2}=r_{0}+\beta$, onde $\beta$ é uma variação próxima de $r_{0}$;

- Para a distribuição centrada em quatro pontos, definimos $r_{1}=r_{0}-2 \beta, r_{2}=r_{0}-\beta, r_{3}=r_{0}+\beta$ e $r_{4}=r_{0}+2 \beta$

- Para a medida de probabilidade distribuída contínua e uniformemente num intervalo $[a, b]$, definimos $a=r_{0}-2 \beta$ e $b=r_{0}+2 \beta$;

-Para a medida de distribuição de Gauss, tomamos o desvio padrão $\sigma=\frac{\beta}{3}$, assim, aproximadamente $97 \%$ das escolhas de $r$ encontram-se dentro de $\left(r_{0}-3 \sigma, r_{0}+3 \sigma\right)$. Desta forma, temos uma probabilidade quase 1 de distribuição dos parâmetros de $r$ para os quais a função exponencial logística apresenta comportamento caótico.

A legenda dos gráficos é definida como:

Solução $\mathrm{L} \Lambda=1$
$\mathrm{~L} \Lambda$ com r variando segundo a distribuição de Dirac
$\mathrm{L} \Lambda$ com r variando segundo a distribuição centrada em dois pontos
$\mathrm{L} \Lambda$ com r variando segundo a distribuição centrada em quatro pontos
$\mathrm{L} \Lambda$ com r variando segundo a distribuição contínua e uniforme no intervalo [a,b]
L $\Lambda$ com r variando segundo a distribuição de Gauss

Figura 1: Legenda dos gráficos das comparações de cada medida de distribuição. 

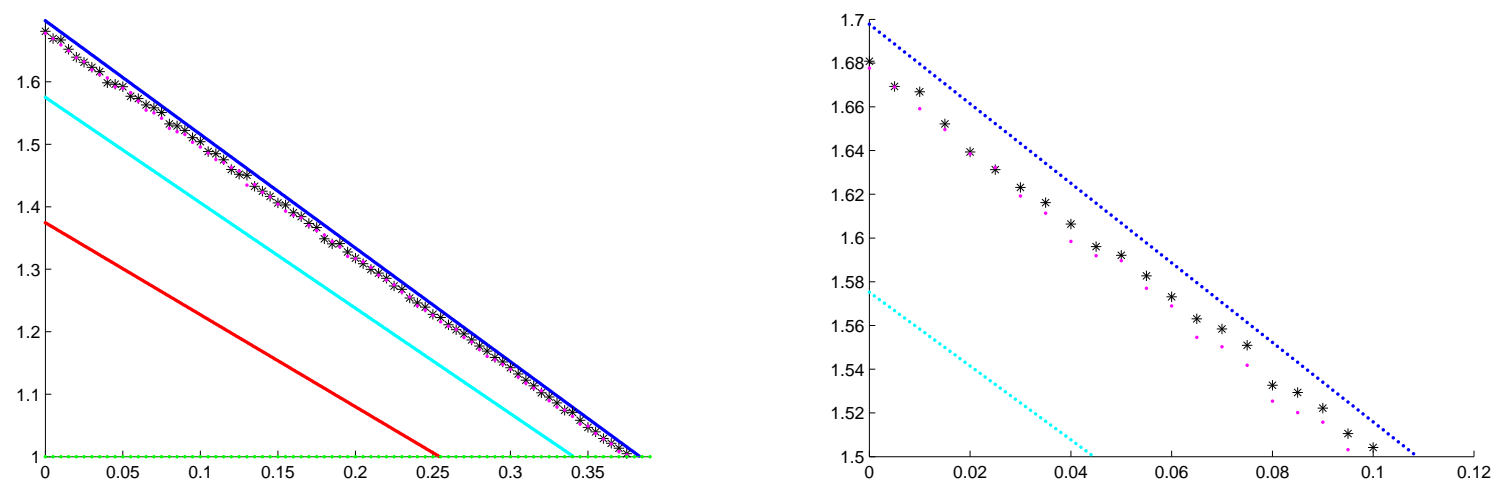

$(a)$
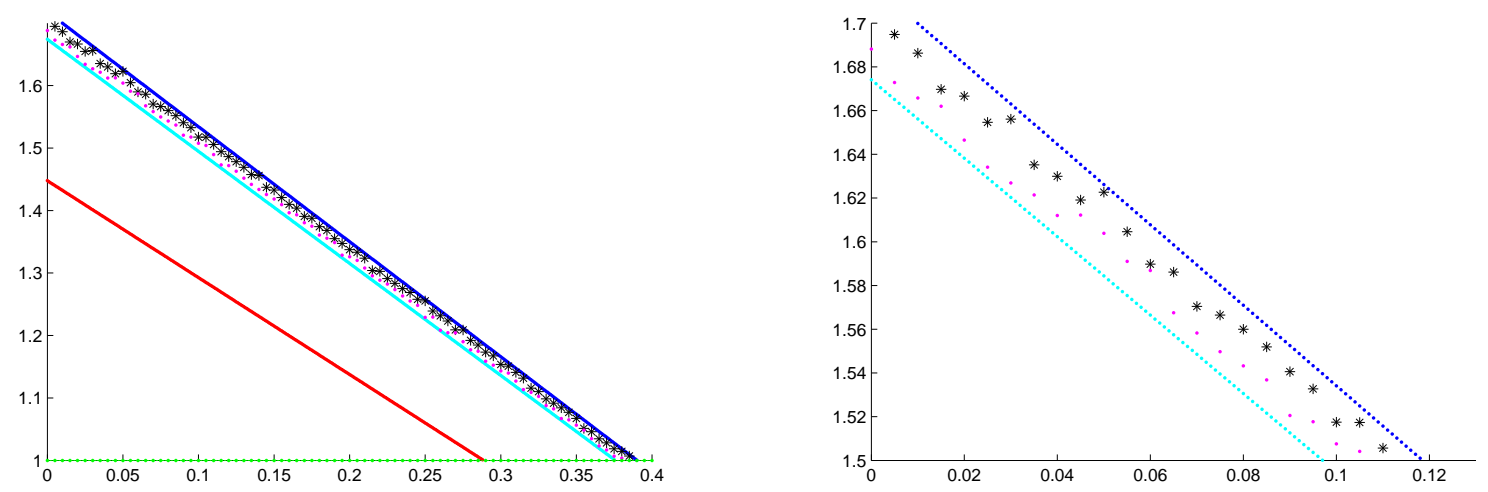

(b)

Figura 2: Gráficos de $L \Lambda \times \mu$ para os 5 exemplos de medida de probabilidade. Acoplamento por Conexão Global com $n=15$. (a) $r_{0}=3.75, \beta=0.05$; (b) $r_{0}=4.3, \beta=0.05$.

Na figura (2) apresentamos os gráficos de $L \Lambda \times \mu$ para os 5 exemplos de medida de probabilidade, com acoplamento por conexão global com o número de sítios $n=15$. A reta constante equivale a $L \Lambda=1$, ou seja, qualquer valor acima dessa reta $(L \Lambda>1)$ indica que nosso sistema não é localmente assintoticamente estável (está na região de impossibilidade de sincronia) para tais valores da fração migratória $\mu$.

Os gráficos do lado direito da figura (2) são ampliações dos gráficos do lado esquerdo, respectivamente. O objetivo dessas ampliações é o de facilitar a comparação entre as medidas de distribuição. Notamos que para valores menores de $\mu$, encontramos impossibilidade de sincronia para todas as medidas de distribuição, enquanto que para valores maiores de taxa migratória isso não ocorre.

Observamos que tanto para $r_{0}=3.75$ (caso (a)) quanto para $r_{0}=4.3$ (caso (b)), utilizando $\beta=0.05$, a distribuição centrada em dois pontos como a distribuição de maior possibilidade de sincronia. Essa distribuição, sincroniza no caso (a) para valores de $\mu>0.245$ e no caso (b) para valores de $\mu>0.3$. Notamos que ao aumentarmos o valor de $r$, obtemos um número de Lyapunov mais expressivo, e por consequência, aumentamos a região de impossibilidade de sincronia.

Ainda investigando a figura (2), no caso (a), a distribuição de $r$ centrada em quatro pontos é a segunda maior região de sincronia. As outras distribuições permanecem com possibilidade de sincronia aleatoriamente próximas. A distribuição de Dirac possui menor possibilidade de sincronia, e a mesma ocorre para $\mu>0.37$, seguida pela distribuição de Gauss e pela distribuição contínua uniforme, respectivamente. No caso (b), exceto a distribuição centrada em dois pontos, 
todas as medidas estão aleatoriamente próximas umas das outras. A medida de Dirac possui menor possibilidade de sincronia, ocorrendo para $\mu>0.385$ seguida pela distribuição de Gauss, pela distribuição contínua e uniforme, e pela distribuição centrada em quatro pontos, sendo essa última, a segunda maior região de possibilidade de sincronia.

\section{Conclusões}

Há um número considerável de estudos sobre a sincronização de metapopulações, como pode ser verificado em Earn [1], Silva [5], entre outros. Segundo Earn [1], o sincronismo está fortemente relacionado com a extinção de metapopulações, desta forma, quanto mais estudos propormos nessa área, maior a chance de encontrarmos resultados mais eficientes para tentar evitá-lo.

Através desses exemplos de medidas de distribuição, ligados a fatores externos, tais como temperatura, precipitação e sazonalidade, temos uma ideia mais realista de como a taxa de reprodução pode variar. Por exemplo, $r$ pode variar em períodos, ou até mesmo variar seguindo uma outra distribuição, por conta da variação desses fatores externos.

O sistema cuja taxa de reprodução intrínseca da população varia através da distribuição centrada em dois pontos possui uma maior possibilidade de sincronização, para todos os parâmetros de $r_{0}$ estudados nas simulações [2]. Portanto, a metapopulação em que varia $r$ através dessa medida possui uma maior possibilidade de sincronização, i.é, esta mais suscetível a extinção global comparada com outras distribuição para esse mesmo sistema. Uma espécie que tem sua taxa de reprodutividade variando no tempo através de dois valores (e.g, inverno e verão), possui uma probabilidade maior de extinção comparada com a mesma espécie seguindo a distribuição de Dirac ( $r$ constate), ou comparada aos outros exemplos de distribuição. Logo, concluímos que a presença do fator externo aumenta a possibilidade de sincronização.

Observamos que há uma probabilidade maior de sincronia para valores menores de parâmetro $r$ e quando o número de sítios aumenta, a sincronização diminui [2]. Concluímos também que aumentos na fração migratória $\mu$ levam a uma maior chance de sincronização, i.é, quanto maior for a fração de indivíduos que migra de um sítio para outros, maior será a possibilidade de sincronia. Esse resultado vai de acordo com os estudos de Earn et al [1]. A migração tem um papel fundamental na sincronização da população, pois quanto mais conexões são estabelecidas entre os sítios, mais propenso à sincronização o sistema se torna [2].

\section{Referências}

[1] EARN, D. J. D.; LEVIN, S. A.; ROHANI, P. Coherence and conservation. Science 290 (2000), 1360-1364.

[2] ESPINOSA, C. E. Sincronização de um Modelo Metapopulacional com a Taxa de Reprodução Intríseca da População Dependente do Tempo. Dissertação de Mestrado, Universidade Federal do Rio Grande do Sul, 2012.

[3] ECKMAN, J. P.; RUELLE, D. Ergodic theory of chaos and strange attractors. Reviews of Modern Physics, v.57, n.3, p.617-656, 1985.

[4] HASSEL, M.P. Density-dependence in single-species populations. J. Anim.,Ecology 44 (1975) 283-295.

[5] SILVA, J. A. L.; GIORDANI, F. T. Density-dependent migration and synchronism in metapopulations. Bull. Math. Biol. 68 (2006), 451-465.

[6] SILVA, J. A. L.; DE CASTRO, M. L.; JUSTO, D. A. R. Synchronism in a metapopulation model. Bull. Math. Biol. 62 (2000), 337-349. 